\title{
Hypertension unawareness among Chinese patients with first-ever stroke
}

\author{
Qinqin Cao ${ }^{1 \dagger}$, Pei Pei ${ }^{2 \dagger}$, Jun Zhang ${ }^{1}$, Jillian Naylor ${ }^{3}$, Xinying Fan ${ }^{1}$, Biyang Cai ${ }^{1}$, Qiliang Dai ${ }^{1}$, Wen Sun ${ }^{1}$, Ruidong Ye \\ Ruifeng Shi ${ }^{1}$, Keting Liu', Yongjun Jiang ${ }^{4}$, Wenhua Liu', Fang Yang ${ }^{1}$, Wusheng Zhu', Yunyun Xiong ${ }^{1}$, \\ Xinfeng Liu ${ }^{1}$ and Gelin $\mathrm{Xu}^{1,4^{*}}$
}

\begin{abstract}
Background: The low rates of hypertension treatment and control, partly due to its unawareness, are the main causes of the high stroke incidence in China. The purpose of this study was to evaluate hypertension unawareness amongst patients with first-ever stroke and to detect factors associated with its unawareness.

Methods: We selected those diagnosed with hypertension from patients with first-ever stroke registered in the Nanjing Stroke Registry Program between 2004 and 2014. These hypertensives were divided as being aware or unaware of their hypertension by using a brief questionnaire conducted shortly after the stroke. Multivariate logistic regression analysis was performed to identify potential factors associated with hypertension unawareness.

Results: Of the 5309 patients with first-ever stroke, 3732 (70.3 \%) were diagnosed with hypertension. Among which, $593(15.9 \%)$ were unaware of their hypertension at the time of stroke onset. Lower-level of education (primary school or illiteracy) and smoking were associated positively with hypertension unawareness; while advanced age, overweight, diabetes mellitus, heart diseases and family history of stroke were associated negatively with hypertension unawareness. Annual data analyzed indicated that the rate of hypertension awareness increased during the past 11 years ( $r=0.613, P=0.045$ for trends).

Conclusions: A substantial proportion (15.9\%) of Chinese patients with hypertension had not been aware of this covert risk until an overt stroke occurred. Hypertension unawareness was associated with lower educational levels and smoking, which address the importance of health education especially in these individuals.
\end{abstract}

Keywords: China, Hypertension unawareness, Patient education, Risk factors, Stroke

\section{Background}

Hypertension is the leading modifiable risk factor for cardiovascular disease, involved in approximately $54 \%$ of stroke cases and $47 \%$ of ischemic heart disease incidences worldwide [1]. With 10-mm Hg lowering in systolic blood pressure (SBP), the relative risk for major cardiovascular events can be reduced by 20 to $25 \%$, with more remarkable effects on stroke than coronary outcomes [2]. Unfortunately, the control rate of hypertension is as low as $5 \%$ in China [3]. This prevalence of

\footnotetext{
* Correspondence: gelinxu@nju.edu.cn

${ }^{\dagger}$ Equal contributors

'Department of Neurology, Jinling Hospital, Medical School of Nanjing University, 305 East Zhongshan Road, Nanjing 210002Jiangsu Province, China ${ }^{4}$ Department of Neurology, Jinling Hospital, Southern Medical University, Nanjing 210002Jiangsu Province, China

Full list of author information is available at the end of the article
}

uncontrolled hypertension has been associated with the high stroke incidence and mortality in developing countries $[2,4]$. Unawareness of elevated blood pressure may be a major contributing factor for the inappropriate control of hypertension, absence of anti-hypertension treatments (including behavioral interventions) and improper monitoring of blood pressure even with treatments. Exploring possible factors associated with unawareness of elevated blood pressure is of vital importance, therefore, for preventing cardiovascular diseases and other hypertension related conditions in the population.

Most of the previous studies on hypertension awareness, treatment and control were conducted in communities or in general populations. Few studies have investigated the hypertension unawareness in stroke patients, especially patients with first-ever stroke. 
Considering the high prevalence of untreated and uncontrolled hypertension in China $[3,5,6]$, it is possible that a substantial proportion of hypertensives have been unaware of their elevated blood pressure until a stroke occurred. It is reasonable to predict that stroke incidence may be reduced if hypertension awareness is strengthened. To evaluate the magnitude of unawareness and elucidate possible correlates, we assessed hypertension unawareness in a large Chinese patient group with first-ever stroke.

\section{Methods}

\section{Study subjects}

Subjects in this study were patients with first-ever stroke enrolled in the Nanjing Stroke Registry Program (NSRP) between January 2004 and December 2014. The NSRP, established in July 2002, is the first hospital-based stroke registry program in the Mainland. Its central base is in Jinling Hospital, Nanjing, a city located in the southeast area of China. Detailed procedures for patient enrollment in NSRP have been published [7]. Patients were eligible for this study if they had a first-ever stroke confirmed by a CT or MRI scan within 14 days of onset, aged 18 years or above, and survived 2 weeks after the index stroke. Written informed consent was obtained from each patient and the study was approved by the Ethics Committee in Jinling Hospital.

\section{Definitions}

Stroke was defined by the World Health Organization (WHO) as rapidly developed clinical signs of focal (or global) disturbance of cerebral function, lasting for more than $24 \mathrm{~h}$ or being interrupted by death within $24 \mathrm{~h}$, with no apparent cause other than of vascular origin [8]. Hypertension was defined as an average SBP $\geq 140 \mathrm{~mm}$ $\mathrm{Hg}$ or an average diastolic blood pressure (DBP) $\geq 90 \mathrm{~mm}$ $\mathrm{Hg}$ on at least three measurements after the first week of stroke onset, or receiving pharmacological treatment for hypertension [9].

\section{Blood pressure monitoring}

To determine the status of blood pressure at time of stroke onset, all patients were scheduled for blood pressure monitoring at least twice daily within 14 days of stroke onset. For those discharged from hospital earlier than the 15th day of stroke onset, blood pressure monitoring continued in local clinics, nursing homes, or at homes by a trained family member with an electronic sphygmomanometer (preferably Omron HEM7051). The results were collected during a subsequent clinical visit. Management of blood pressure in patients with acute stroke was in accordance with guidelines. For example, the blood pressure should not be lowered in the acute stage of stroke unless it is higher than 220/ $120 \mathrm{mmHg}$ [10].

\section{Evaluation of blood pressure awareness}

To evaluate the awareness of blood pressure status, each patient enrolled was assessed with a brief questionnaire shortly after stroke admission. Issues in the questionnaire included experiences and frequency of blood pressure measurement before the index stroke, numeric SBP and DBP if measured, awareness of a hypertension diagnosis raised by a physician, any antihypertensive medication usage within 2 weeks before the index stroke, blood pressure responsiveness to the treatments and history of cardiovascular disease other than stroke. The questionnaire was preferably responded by the patient. In case of coma or advanced cognitive impairment, a relative or guardian who was familiar with the medical history of the patient was encouraged to participate in the questionnaire survey. To exclude possible memory bias, the survey was conducted before blood pressure monitoring.

\section{Stratification of blood pressure status}

For all patients, results of blood pressure monitoring and history of antihypertensive medication were considered for the diagnosis of hypertension at time of stroke onset. Patients diagnosed with hypertension were grouped as being aware or unaware of this condition based on their responses to the questionnaire. Those who were aware of the hypertension were stratified as treated or untreated, and those being treated were further stratified as controlled or uncontrolled. Being aware of hypertension indicated that the patient or family members recalled a hypertension diagnosis made by a physician. Being treated for hypertension indicated that the patient took at least one antihypertensive medication for elevated blood pressure within 2 weeks immediately before the index stroke $[3,6,11]$. When the DBP $<90 \mathrm{~mm}$ $\mathrm{Hg}$ and the SBP $<140 \mathrm{~mm} \mathrm{Hg}$, hypertension was regarded as being controlled [12].

\section{Possible influencing factors}

Data of demographic profiles (age, sex, marriage status), major risk factors for cardiovascular disease other than hypertension (diabetes mellitus (DM), hyperlipidaemia, smoking, alcohol drinking, overweight), type of index stroke, history of heart diseases, socioeconomic status (resident environment, occupation, educational level, possession of health insurance) and family history of stroke were collected from NSRP and analyzed as potential influencing factors associated with hypertension unawareness. Smoking was defined as that patient had smoked more than 1 cigarette per day for at least 6 months. Alcohol drinking was defined as that patient consumed more than two drinks a day for at least 
2 months. Overweight was defined as a body mass index (BMI) $\geq 25 \mathrm{~kg} / \mathrm{m}^{2}$. Presences of DM, heart diseases and hyperlipidaemia were confirmed by medical records.

\section{Statistical analysis}

Kolmogorov-Smirnov test was performed to determine whether the distribution of a continuous parameter was normal. Continuous variables were presented as median (interquartile range $[\mathrm{IQR}]$ ), whilst categorical variables were presented as frequencies. For inter-group comparisons, continuous variables were analyzed with MannWhitney $U$ test, whilst categorical variables were analyzed with $\chi^{2}$ test or Fisher's exact test. To identify possible factors associated with hypertension unawareness, the significant variables $(P<0.1)$ identified in univariate analyses were entered in a multivariate logistic regression model for further analysis. Results were presented as odds ratio (OR) and $95 \%$ confidence interval (CI). The linear regression analysis was performed to test the temporal trends of hypertension awareness, treatment and control over years. $P<0.05$ for two-sided test was deemed as statistical significance. All analyses were conducted using SPSS software version 17.0 (SPSS Inc, Chicago, IL).

\section{Results}

The study screened 5309 patients with first-ever stroke registered in NSRP between 2004 and 2014. Based on the results of blood pressure monitoring and history of antihypertensive medication usage, 3732 (70.3 \%) patients were confirmed with hypertension at time of stroke onset. These 3732 patients were selected as subjects for the analyses of hypertension unawareness and the factors associated.

Of all 3732 hypertensive patients chosen, 3367 $(90.2 \%)$ were diagnosed with ischemic stroke, 222 $(6.0 \%)$ with intracerebral hemorrhage and 143 (3.8 \%) with subarachnoid hemorrhage. At time of stroke onset, 3139 (84.1\%) patients were aware of their hypertension through a previous diagnosis, whilst 593 (15.9\%) were unaware; 1534 (41.1\%) received anti-hypertension treatment, and $658(17.6 \%)$ had their blood pressure controlled.

After dichotomizing patients as being aware or unaware of hypertension at time of stroke onset, data of demographic profiles, major risk factors for stroke, socioeconomic status and family history of stroke from each group were compared using univariate analysis (Table 1). In contrast with patients who were aware of their hypertension, those who were unaware were younger (median 60 vs 62 years, $P=1.31 \times 10^{-4}$ ) and had a lower BMI (median 24.0 vs $24.5, P=3.47 \times 10^{-5}$ ). They also had a lower prevalence of DM (13.7\% vs $27.8 \%$, $\left.P=4.34 \times 10^{-14}\right)$, heart diseases $(5.4 \%$ vs $11.0 \%, P=$ $\left.1.44 \times 10^{-5}\right)$ and family history of stroke $(4.6 \%$ vs $11.1 \%$, $\left.P=1.55 \times 10^{-7}\right)$. Furthermore, patients who were smokers (42.0\% vs $35.5 \%, P=0.003)$, engaged in manual work $\left(62.6 \%\right.$ vs $\left.53.2 \%, P=2.78 \times 10^{-5}\right)$, lack of health insurance (55.0 \% vs $49.8 \%, P=0.022)$, with lower-level of education

Table 1 Baseline characteristics of stroke patients by hypertension awareness

\begin{tabular}{|c|c|c|c|}
\hline & Aware $(n=3139)$ & Unaware $(n=593)$ & $P$ value \\
\hline Age, median (IQR) & $62(54-70)$ & $60(50-69)$ & $1.31 \times 10^{-4}$ \\
\hline Age $\geq 60$ years, $n(\%)$ & $1835(58.5)$ & $300(50.6)$ & $4.14 \times 10^{-4}$ \\
\hline Male, n (\%) & $2186(69.6)$ & $412(69.5)$ & 0.961 \\
\hline Married, n (\%) & 3135 (99.9) & $592(99.8)$ & 0.579 \\
\hline BMI, median (IQR) & $24.5(22.9-26.0)$ & $24.0(22.5-25.4)$ & $3.47 \times 10^{-5}$ \\
\hline $\mathrm{BMI} \geq 25, \mathrm{n}(\%)$ & $1242(39.6)$ & $198(33.4)$ & 0.005 \\
\hline Smoking, n (\%) & $1115(35.5)$ & $249(42.0)$ & 0.003 \\
\hline Alcohol drinking, n (\%) & $682(21.7)$ & $142(23.9)$ & 0.235 \\
\hline Diabetes mellitus, n (\%) & $874(27.8)$ & $81(13.7)$ & $4.34 \times 10^{-14}$ \\
\hline Hyperlipidaemia, n (\%) & $469(14.9)$ & $76(12.8)$ & 0.205 \\
\hline Heart diseases, n (\%) & $345(11.0)$ & $32(5.4)$ & $1.44 \times 10^{-5}$ \\
\hline History of TIA, n (\%) & $123(3.9)$ & $18(3.0)$ & 0.348 \\
\hline Lack of health insurance, n (\%) & $1564(49.8)$ & $326(55.0)$ & 0.022 \\
\hline Rural residency, n (\%) & $877(27.9)$ & $228(38.4)$ & $5.23 \times 10^{-7}$ \\
\hline Lower-level of education, n (\%) & $806(27.3)$ & $222(38.0)$ & $3.35 \times 10^{-7}$ \\
\hline Manual work, n (\%) & $1663(53.2)$ & $371(62.6)$ & $2.78 \times 10^{-5}$ \\
\hline Family history of stroke, $n$ (\%) & $349(11.1)$ & $27(4.6)$ & $1.55 \times 10^{-7}$ \\
\hline
\end{tabular}

$I Q R$ indicates interquartile ranges, $B M I$ indicates body mass index, $T I A$ indicates transient ischemic attack. Lower-level of education indicates primary school or illiteracy 
(primary school or illiteracy, $38.0 \%$ vs $27.3 \%, P=3.35 \times$ $\left.10^{-7}\right)$, and lived in rural areas (38.4\% vs $27.9 \%, P=5.23 \times$ $10^{-7}$ ) were less likely to be aware of their hypertension.

To determine potential factors attributable to hypertension unawareness, those parameters manifested significant difference $(P<0.1)$ between groups were further analyzed with multivariate logistic regression (Table 2). Smokers (OR: 1.38 ; 95 \% CI: 1.11 to 1.71 ) and those with lower-level of education (OR: 1.61; 95 \% CI: 1.27 to 2.03) were less likely to be aware of their hypertension. Whilst patients with advanced age (OR: 0.69; $95 \% \mathrm{CI}: 0.56$ to 0.83), overweight (OR: 0.77; $95 \% \mathrm{CI}: 0.64$ to 0.94 ), DM (OR: $0.44,95 \% \mathrm{CI}: 0.34$ to 0.57 ), heart diseases (OR: $0.54,95 \% \mathrm{CI}: 0.37$ to 0.80 ) or with family history of stroke (OR: $0.38,95 \%$ CI: 0.25 to 0.57 ) were more likely to be aware of their hypertension.

To assess the temporal trends of hypertension awareness, treatment, and control, we evaluated these rates over the years (Fig. 1). Linear regression indicated the rate of awareness increased slightly but significantly in 11 years (from $84.0 \%$ in 2004 to $87.4 \%$ in 2014, $r=$ $0.613, P=0.045$ for trends). Although rates of hypertension treatment (from $30.8 \%$ in 2004 to $51.9 \%$ in 2014, $r=0.947, P=9.89 \times 10^{-6}$ for trends) and control (from $9.0 \%$ in 2004 to $25.3 \%$ in 2014, $r=0.890, P=2.39 \times$ $10^{-4}$ for trends) at time of stroke onset increased, there was considerable margin for improvement due to the extensive hypertension unawareness.

\section{Discussion}

This study presents several important findings regarding hypertension unawareness, associated factors as well as hypertension treatment and control in China. A substantial proportion (15.9\%) of Chinese patients with hypertension

Table 2 Factors associated with hypertension unawareness

\begin{tabular}{lll}
\hline \multirow{2}{*}{ Variables } & \multicolumn{2}{l}{ Hypertension unawareness } \\
\cline { 2 - 3 } Age $\geq 60 y$ & $0.69(0.56$ to 0.83$)$ & 0.0002 \\
Male & $0.93(0.73$ to 1.17$)$ & 0.518 \\
BMI $\geq 25$ & $0.77(0.64$ to 0.94$)$ & 0.009 \\
Smoking & $1.38(1.11$ to 1.71$)$ & 0.004 \\
Heart diseases & $0.54(0.37$ to 0.80$)$ & 0.002 \\
Diabetes mellitus & $0.44(0.34$ to 0.57$)$ & $2.53 \times 10^{-10}$ \\
Lack of health insurance & $0.93(0.75$ to 1.15$)$ & 0.489 \\
Rural residency & $1.23(0.96$ to 1.57$)$ & 0.103 \\
Lower-level of education & $1.61(1.27$ to 2.03$)$ & $0.73 \times 10^{-4}$ \\
Manual work & $1.13(0.90$ to 1.41$)$ & 0.282 \\
Family history of stroke & $0.38(0.25$ to 0.57$)$ & $2.58 \times 10^{-6}$ \\
\hline
\end{tabular}

Parameters were analyzed with multivariate logistic regression analysis. $O R$ indicates odds ratio, $\mathrm{Cl}$ indicates confidence interval, $\mathrm{BMI}$ indicates body mass index had not been aware of this covert risk until an overt stroke occurred. Lower-level of education and smoking were associated with hypertension unawareness, whilst advanced age, overweight, DM, heart diseases and family history of stroke were associated with improved hypertension awareness.

As expected, this study associated lower-level of education (primary school or illiteracy) with hypertension unawareness in Chinese stroke patients. Patients with lower-level of education may lack sufficient drive and/or adequate skills to monitor their blood pressure over an extended period of time [13-15]. Their knowledge of hypertension as a risk factor for cardiovascular disease may be limited compared with that of those with advanced education [16-18]. They are more prone to disregard health education programs as available [13]. Poorly educated patients, therefore, may be less likely to have their blood pressure measured regularly [16]. Conversely, the promoting effects of advanced education on hypertension awareness have been observed in other studies $[11,19]$.

In this study, hypertension unawareness was more prevalent in young than in elderly patients. Young people may be less vigilant to the hazardous effects of elevated blood pressure to their health. Many other studies also reported that young people have a lower rate of hypertension awareness [11, 19-21].

Compared with non-smoking patients, smokers were less likely to be aware of their hypertension. This finding was in consistent with results from some communitybased studies [20, 22], but contrary to those from others $[11,19]$. This finding highlighted the importance of hypertension education in smoking individuals, which may be more feasible and effective in communities. Still other studies have reported that women had a higher level of hypertension awareness than men, however in this study we did not detect different hypertension awareness between sexes $[11,19,20]$. Selection bias may be responsible for these discrepancies, for example, stroke patients enrolled in this study showed a male predominance (69.6\%), while the sex distribution of community populations were more even. No significant correlation between health insurance and hypertension unawareness was detected in this study. The socioeconomic development was remarkable in recent years, but the situation that patients are responsible for their own health remained largely unchanged in Nanjing and also in China. Although more citizens especially those in urban were covered by the government-run health insurance in recent 10 years, there was no regular blood pressure-checking plan even for patients with hypertension. Patients, with or without health insurance, usually had their blood pressure being checked at their own will. 


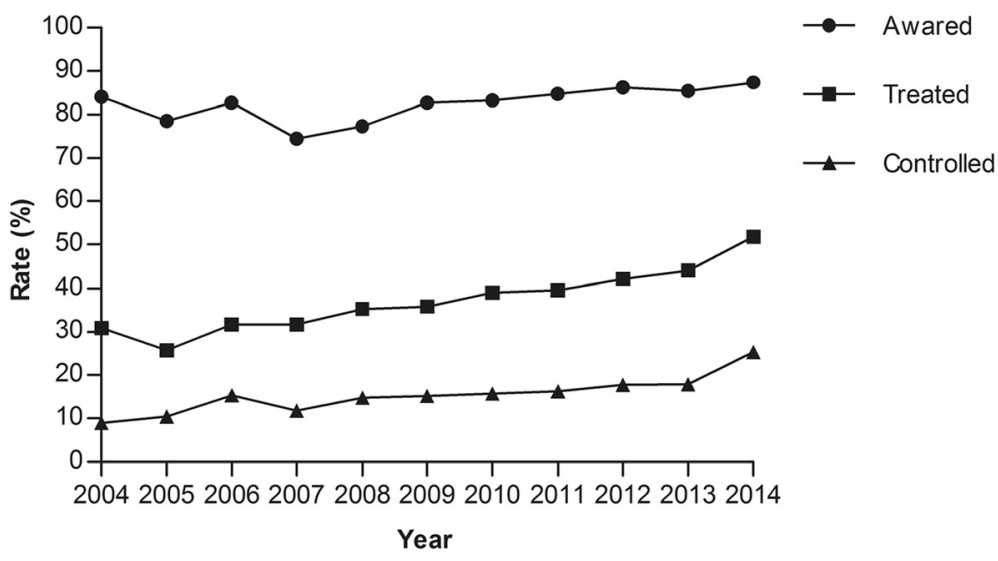

Fig. 1 Trends in awareness, treatment, and control of hypertension in stroke patients, 2004-2014. There had been significant increases in hypertension awareness ( $r=0.613, P=0.045$ for trends), treatment $\left(r=0.947, P=9.89 \times 10^{-6}\right.$ for trends), and control $\left(r=0.890, P=2.39 \times 10^{-4}\right.$ for trends) in this stroke patient cohort during the 11 years

In this study, patients with comorbidities, such as DM, heart diseases and overweight were more likely to be aware of their hypertension. These concomitant chronic conditions usually require regular follow-ups, thus the occasions of patients meeting with their healthcare providers may increase, so does the chance of a covert hypertension being diagnosed. Patient's experiences in diagnosing and managing DM, heart diseases and overweight may help for improving blood pressure administration. Other chronic diseases may also alert the patients with the possible catastrophic outcomes of uncontrolled hypertension, therefore reinforcing their drive for blood pressure monitoring and management. These synergetic effects of concomitant chronic diseases on hypertension management were also reported in other studies [19, 20, 22].

Several major limitations of the study should be emphasized when interpreting the results. Responsive elevation of blood pressure was not uncommon after acute stroke especially in hemorrhagic stroke [23]. The blood pressure may rise as a result of increased intracranial pressure, pain, infection, psychological stress, urine retention, damage of brain regions that regulate the autonomic nerve tonic after stroke [24]. These responsive hypertensions were usually transient and the blood pressure, in most cases, would return to the baseline levels within the first week $[25,26]$. To prevent misdiagnosed hypertension due to these conditions, blood pressure was monitored at least twice daily within 14 days of stroke onset. Because only $6 \%$ of enrolled stroke patients were hemorrhagic in this study, we believe the total impact of inconsistency between pre-and post-stroke blood pressure in hemorrhagic stroke was minimal. In addition, hypotension caused by the index stroke (e.g., infarct in insula) may increase the likelihood of false negative in presuming a hypertension diagnosis immediately before the index stroke. But this condition is less common [27].
Cognitive impairments were common in the first few weeks after stroke [28]. Impaired consciousness may also present in stroke patients [29]. Both conditions may affect the evaluation of hypertension awareness at time of stroke onset. To minimize these confounding effects, we asked a relative or guardian who was familiar with the medical history of the patient to confirm the hypertension awareness before the index stroke. The questionnaire was conducted before blood pressure monitoring to exclude possible memory bias.

Finally, all patients in this study were enrolled from one center (Nanjing) in southeast China, where the socioeconomic development is noticeably more advanced, educational level higher and general healthcare status more advantageous than other parts of the country. Thus, the awareness of hypertension may be higher in this patient group than that in other populations.

\section{Conclusions}

A substantial proportion (15.9 \%) of Chinese patients with hypertension had not been aware of this covert risk until an overt stroke occurred. Hypertension unawareness was associated with lower educational levels and smoking, which address the importance of health education especially in these individuals.

\section{Abbreviations \\ SBP: systolic blood pressure; NSRP: Nanjing Stroke Registry Program: WHO: World Health Organization; DBP: diastolic blood pressure; DM: diabetes mellitus; BMI: body mass index; IQR: interquartile range; OR: odds ratio; $\mathrm{Cl}$ : confidence interval.}

Competing interests

The authors declare that they have no competing interests.

Authors' contributions

GX conceived and designed the study. QC wrote the draft of the manuscript. $X F, R Y, R S, K L, Y J, W L, F Y, W Z$ and $Y X$ collected the data. BC, $Q D$ and WS undertook the statistical analyses. PP, JZ and JN gave critical comments on 
the draft and contributed to the manuscript writing. GX and XL supervised this study and approved of the version to be submitted. All authors read and approved the final manuscript.

\section{Acknowledgements}

This study was supported partly by National Natural Science Foundation of China (NSFC 81301017 to XF, NSFC 81171099 to WZ), the Jiangsu Nature Science Foundation (BE2013713 to GX, BK2010067 to WZ). The study was conducted in the department of Neurology, Jinling Hospital, which provided core support for this work. We thank all the participants of this study and research nurses, interviewers and other staff who have taken part in gathering or analyzing the data of this study.

\section{Author details}

${ }^{1}$ Department of Neurology, Jinling Hospital, Medical School of Nanjing University, 305 East Zhongshan Road, Nanjing 210002Jiangsu Province, China. ${ }^{2}$ School of Humanity, Ningbo Dahongying University, Ningbo 315175Zhejiang Province, China. ${ }^{3}$ Department of Neurology, Melbourne Brain Centre, Royal Melbourne Hospital, University of Melbourne, Melbourne, Victoria, Australia. ${ }^{4}$ Department of Neurology, Jinling Hospital, Southern Medical University, Nanjing 210002Jiangsu Province, China.

Received: 31 October 2015 Accepted: 4 February 2016 Wer. -

\section{References}

1. Lawes CM, Vander Hoorn S, Rodgers A. Global burden of blood-pressurerelated disease, 2001. Lancet. 2008;371:1513-8.

2. Perkovic V, Huxley R, Wu Y, Prabhakaran D, MacMahon S. The burden of blood pressure-related disease: a neglected priority for global health. Hypertension. 2007;50:991-7.

3. Wu Y, Huxley R, Li L, Anna V, Xie G, Yao C, et al. Prevalence, awareness, treatment, and control of hypertension in China: data from the China National Nutrition and Health Survey 2002. Circulation. 2008;1 18:2679-86.

4. Ibrahim MM, Damasceno A. Hypertension in developing countries. Lancet. 2012;380:611-9.

5. Gu D, Reynolds K, Wu X, Chen J, Duan X, Muntner P, et al. Prevalence, awareness, treatment, and control of hypertension in china. Hypertension. 2002:40:920-7

6. Li D, Lv J, Liu F, Liu P, Yang X, Feng Y, et al. Hypertension burden and control in mainland China: Analysis of nationwide data 2003-2012. Int J Cardiol. 2015;184:637-44.

7. Liu X, Xu G, Wu W, Zhang R, Yin Q, Zhu W. Subtypes and one-year survival of first-ever stroke in Chinese patients: The Nanjing Stroke Registry. Cerebrovasc Dis. 2006;22:130-6.

8. Aho K, Harmsen P, Hatano S, Marquardsen J, Smirnov VE, Strasser T. Cerebrovascular disease in the community: results of a WHO collaborative study. Bull World Health Organ. 1980;58:113-30.

9. The sixth report of the Joint National Committee on prevention, detection evaluation, and treatment of high blood pressure. Arch Intern Med. 1997; 157:2413-46.

10. Adams Jr HP, Adams RJ, Brott T, del Zoppo GJ, Furlan A, Goldstein LB, et al. Guidelines for the early management of patients with ischemic stroke: A scientific statement from the Stroke Council of the American Stroke Association. Stroke. 2003;34:1056-83.

11. Muntner P, Gu D, Wu X, Duan X, Wenqi G, Whelton PK, et al. Factors associated with hypertension awareness, treatment, and control in a representative sample of the chinese population. Hypertension. 2004;43:578-85.

12. Chobanian AV, Bakris GL, Black HR, Cushman WC, Green LA, Izzo Jr JL, et al. The Seventh Report of the Joint National Committee on Prevention, Detection, Evaluation, and Treatment of High Blood Pressure: the JNC 7 report. JAMA. 2003;289:2560-72.

13. Winkleby MA, Jatulis DE, Frank E, Fortmann SP. Socioeconomic status and health: how education, income, and occupation contribute to risk factors for cardiovascular disease. Am J Public Health. 1992;82:816-20.

14. Tedesco MA, Di Salvo G, Caputo S, Natale F, Ratti G, larussi D, et al. Educational level and hypertension: how socioeconomic differences condition health care. J Hum Hypertens. 2001;15:727-31.

15. Liu Q, Wang M, Guo J, Li J, Li C, Qian M. Effect of socioeconomic status on secondary prevention of stroke. Int J Qual Health Care. 2011;23:405-12.
16. Samal D, Greisenegger S, Auff E, Lang W, Lalouschek W. The relation between knowledge about hypertension and education in hospitalized patients with stroke in Vienna. Stroke. 2007;38:1304-8.

17. Wang Z, Yue X, Wang H, Bao C, Xu W, Chen L, et al. Relation of socioeconomic status to hypertension occurrence. Int J Cardiol. 2014;173:544-5.

18. Leng $B$, Jin $Y$, Li G, Chen L, Jin N. Socioeconomic status and hypertension: a meta-analysis. J Hypertens. 2015;33:221-9.

19. Jaddou HY, Batieha AM, Khader YS, Kanaan AH, El-Khateeb MS, Ajlouni KM. Hypertension prevalence, awareness, treatment and control, and associated factors: results from a national survey, jordan. Int J Hypertens. 2011;2011:828797.

20. Wyatt SB, Akylbekova EL, Wofford MR, Coady SA, Walker ER, Andrew ME, et al. Prevalence, awareness, treatment, and control of hypertension in the Jackson Heart Study. Hypertension. 2008:51:650-6.

21. Wu Y, Tai ES, Heng D, Tan CE, Low LP, Lee J. Risk factors associated with hypertension awareness, treatment, and control in a multi-ethnic Asian population. J Hypertens. 2009;27:190-7.

22. Mendez-Chacon E, Santamaria-Ulloa C, Rosero-Bixby L. Factors associated with hypertension prevalence, unawareness and treatment among Costa Rican elderly. BMC Public Health. 2008:8:275.

23. Fischer U, Cooney MT, Bull LM, Silver LE, Chalmers J, Anderson CS, et al. Acute post-stroke blood pressure relative to premorbid levels in intracerebral haemorrhage versus major ischaemic stroke: a populationbased study. Lancet Neurol. 2014;13:374-84.

24. Qureshi Al. Acute hypertensive response in patients with stroke: pathophysiology and management. Circulation. 2008;1 18:176-87.

25. Semplicini A, Maresca A, Boscolo G, Sartori M, Rocchi R, Giantin V, et al. Hypertension in acute ischemic stroke: a compensatory mechanism or an additional damaging factor? Arch Intern Med. 2003;163:211-6.

26. Toyoda K, Okada Y, Fujimoto S, Hagiwara N, Nakachi K, Kitazono T, et al. Blood pressure changes during the initial week after different subtypes of ischemic stroke. Stroke. 2006;37:2637-9.

27. Robinson TG, Potter JF. Blood pressure in acute stroke. Age Ageing. 2004;33:6-12.

28. Nys GM, van Zandvoort MJ, de Kort PL, Jansen BP, de Haan EH, Kappelle LJ. Cognitive disorders in acute stroke: prevalence and clinical determinants. Cerebrovasc Dis. 2007;23:408-16.

29. Edlow JA, Selim MH. Atypical presentations of acute cerebrovascular syndromes. Lancet Neurol. 2011;10:550-60.

\section{Submit your next manuscript to BioMed Central and we will help you at every step:}

- We accept pre-submission inquiries

- Our selector tool helps you to find the most relevant journal

- We provide round the clock customer support

- Convenient online submission

- Thorough peer review

- Inclusion in PubMed and all major indexing services

- Maximum visibility for your research

Submit your manuscript at www.biomedcentral.com/submit
Biomed Central 